\title{
Transfer of two Indian Idiops spp. to the genus Heligmomerus Simon, 1892 (Araneae: Idiopidae) with redescription of $\boldsymbol{H}$. barkudensis (Gravely, 1921)
}

\author{
Manju Siliwal ${ }^{1,2}$, Sanjay Molur ${ }^{1,3} \&$ Robert Raven ${ }^{4}$ \\ 1,2 Wildlife Information Liaison Development Society / ${ }^{3}$ Zoo Outreach Organisation, \\ 9-A, Lal Bahadur Colony, Peelamedu, Coimbatore, Tamil Nadu 641004, India \\ ${ }^{4}$ Queensland Museum, Grey Street, PO Box 3300, South Brisbane, 4101, Queensland, Australia \\ Email: ${ }^{2}$ manjusiliwal@gmail.com, ${ }^{3}$ herpinvert@gmail.com, ${ }^{4}$ RobertR@qm.qld.gov.au
}

Date of publication (online): 26 June 2010 Date of publication (print): 26 June 2010 ISSN 0974-7907 (online) | 0974-7893 (print)

Editor: Ansie Dippenaar-Schoeman

\section{Manuscript details:}

Ms \# 02344

Received 11 November 2009

Final revised received 27 February 2010

Finally accepted 15 May 2010

Citation: Siliwal, M., S. Molur \& R. Raven (2010). Transfer of two Indian Idiops spp. to the genus Heligmomerus Simon, 1892 (Araneae: Idiopidae) with redescription of $H$. barkudensis (Gravely, 1921). Journal of Threatened Taxa 2(6): 940-947.

Copyright: (C) Manju Siliwal, Sanjay Molur \& Robert Raven 2010. Creative Commons Attribution 3.0 Unported License. JoTT allows unrestricted use of this article in any medium for non-profit purposes, reproduction and distribution by providing adequate credit to the authors and the source of publication.

Author Details: MANJU SILIWAL is an arachnologist working with Wildlife Information Liaison Development Society since 2002. She is working on ecology, taxonomy and conservation of mygalomorph spiders of India. SANJAY MoluR is the Founder Secretary of WILD and Executive Director of ZOO. He is currently involved in studies on rodents and bats in Coorg. He is also involved in trade and ecological studies of mygalomorph spiders in India since 2000. Robert Raven is Head of Terrestrial Biodiversity at Queensland Museum. $\mathrm{He}$ is one of the leading arachnologists of the world. His main interest lies in studying mygalomorph spiders.

Author Contribution, Acknowledgements: See end of this article.

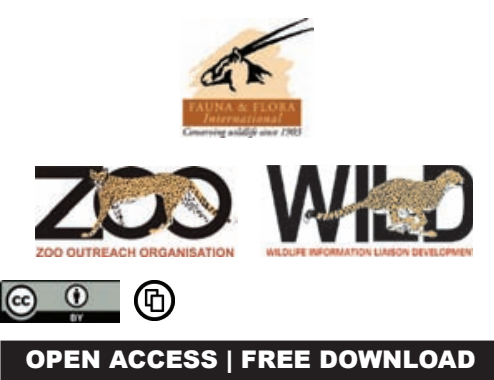

Abstract: The genus Heligmomerus Simon, 1892 of the trapdoor spider family Idiopidae is represented by a single species, $H$. prostans Simon, 1892 in India. Idiops biharicus Gravely, 1915 and I. barkudensis Gravely, 1921 were found to be misplaced in the genus Idiops and transfer to the genus Heligmomerus based on a dorsal saddle-shape depression on tibiae III. The Heligmomerus barkudensis (Gravely, 1921) was previously known only from the type locality but during the recent surveys in Orissa it was sampled from several locations on the mainland in Orissa as well as other islands of Chilika Lake. $H$. barkudensis is redescribed and natural history notes are provided based on the new specimen collections from the type locality, Barkuda Island and other new localities in Orissa.

Keywords: Heligmomerus, Idiops, taxonomy, trapdoor spiders.

\section{INTRODUCTION}

The family Idiopidae is represented by 22 genera and 303 species in three subfamilies in the world (Platnick 2010). The members of the subfamily Idiopinae can be identified by having anterior lateral eyes situated close to the clypeal edge, well advanced from the rest of the eye group (Raven 1985). So far, only two genera of the Idiopidae, Idiops Perty, 1833 and Heligmomerus Simon, 1892 have been reported from India (Siliwal \& Molur 2007; Platnick 2010).

The genus Heligmomerus is an old world genus and so far eight species are known, mainly from Africa (Dippenaar-Schoeman 2002; Platnick 2010); two species have been reported from South Asia, H. taprobanicus Simon, 1892 from Sri Lanka and $H$. prostans Simon, 1892 from India (Siliwal \& Molur 2007; Platnick 2010). Both species are known only from the type locality. The description of both the species was based on a single specimen and a few morphological characters were described, hampering comparative work. Since Simon (1892) designated a juvenile spider as the type specimen of $H$. taprobanicus, there is a need for collection of more information on systematics of the genus Heligmomerus from this region.

During theraphosid spider surveys in Orissa in March-April 2007, many trapdoor spiders were collected from Dhenkenal, Puri, Sambalpur and Ganjam districts, all having a dorsal depression on tibia III with the anterior lateral eyes situated close to the clypeal edge. On closer examination, they were identified as Heligmomerus spp. belonging to the subfamily Idiopinae of the

Abbreviations: ALE - anterior lateral eye; AME - anterior median eye; MOQ - median ocular quadrate; PLE - posterior lateral eye; PME - posterior median eye; PLS - posterior later spinnerets; PMS - posterior median spinnerets; WILD - Wildlife Information Liaison Development Society. Abbreviations used for hair and spines count are $d$ - dorsal; fe - femur; mt - metatarsus; $p$ - prolateral; pa - patella; $r$ - retrolateral; ta - tarsus; ti - tibia; $v$ - ventral.

Journal of Threatened Taxa I www.threatenedtaxa.org I June 2010 I 2(6): 940-947 
family Idiopidae (Cambridge 1890; Pocock 1900; Raven 1985). Initially, they were considered unidentified species as many characters did not match the description of $H$. prostans. However on reviewing the literature, it was found that Gravely (1935) had proposed the transfer of Idiops biharicus Gravely, 1915 and I. barkudensis Gravely, 1921 to the genus Heligmomerus based on the presence of a saddle-shaped dorsal depression on tibia III. This note was missed during compilation of various spider checklists and updates (Siliwal et al. 2005; Siliwal \& Molur 2007; Platnick 2010). This necessitated comparison of specimens from the mainland with Idiops barkudensis from Barkuda Island as the original description of the species lacks information on spermathecae structures (Gravely 1921). One female and a few immature individuals of Heligmomerus spp. were collected from Barkuda Island and a few specimens from Sanakuda and Kalijai Islands in the Chilika Lake. Examinatation of the spermathecal structure revealed that all specimens belong to the same species. The specimens from Barkuda Island were confirmed to be Idiops barkudensis as noted by Gravely (1935), they had a saddle-shaped depression dorsally on tibia III. With this we validate the transfer of Idiops barkudensis to the genus Heligmomerus as previously proposed by Gravely (1935). The other specimens of Heligmomerus from the mainland of Orissa and other islands were confirmed to belong to $H$. barkudensis based on the spermathecal structure.

The type specimens of Idiops biharicus at the Zoological Survey of India, Kolkata could not be located but based on the description of Gravely (1935), Idiops biharicus is transferred to the genus Heligmomerus. However, re-examination of the type specimens for all the known species of Idiops is recommended to validate their taxonomic position as information about the depression on tibia III is not always provided.

In this paper, we provide additional morphological characters for $H$. barkudensis along with illustrations and natural history notes.

\section{METHODS}

Measurements of body parts except for the eyes were taken with a Mitutoyo ${ }^{\mathrm{TM}}$ Vernier Caliper. Eye measurements were done with a calibrated ocular micrometer. All measurements are in $\mathrm{mm}$. Spermathecae were dissected and cleaned in concentrated lactic acid in $100^{\circ} \mathrm{C}$ water bath for $15-20$ minutes. All illustrations were prepared with the help of camera lucida attached to a CETIIM stereomicroscope by MS. Variations in measurements for female specimens are given as a range (Mean \pm SD). All specimens are deposited at the Wildlife Information Liaison Development Society, Coimbatore, Tamil Nadu.

\section{Heligmomerus Simon, 1892}

Heligmomerus Simon, 1892: 90-91; Pocock, 1900:164; Raven, 1985: 138

Type: Heligmomerus taprobanicus Simon, 1892 based on an immature individual. Not examined.

Diagnosis: This genus differs from the genus Idiops by having a smooth saddle-shaped depression on the basal half of tibia III (Pocock 1900; Raven 1985).

Distribution: Africa, India, Sri Lanka.

\section{Heligmomerus barkudensis (Gravely, 1921) comb. nov.}

Figures 1-10, Images 1-9

Idiops barkudensis Gravely 1921: 399-401

Material examined: 1 female, 18.viii.2007, Chilika Lake, Barkuda Island, Orissa, India, 19033'15.1'N \& 85 08'45.5”E, 113m, coll. S. Behera, M. Siliwal \& G. Sahu (WILD-07-ARA-191); 2 females, 2 immature, 7.iv.2007, Kapilas temple campus, Dhenkanal District, $20^{\circ} 40^{\prime} 54.6^{\prime \prime} \mathrm{N}$ \& 85045'58.4"E, 568m, coll. M. Siliwal and S. Behera, (WILD-07-ARA-171, 172, 173, 174); 1 female, 1 subadult female, 17.iv.2007, near Berbera-Dhuanali Reserve Forest, Balugaon, Puri District, coll. S. Behera, S. Kumar and M. Siliwal (WILD-07-ARA-175, 176); 1 immature, 15.iv.2007, Jacum check post, periphery of Karlapat Wildlife Sanctuary, 263m, 19044'57.5”N \& 8306'38.7"E, coll. S. Behera, S. Kumar and M. Siliwal (WILD-07ARA-181); 2 females, 1 immature, Kalijai Island, Chilika Lake, 19³9'55.8”N \& 85012'55.2"E, 106m, coll. S. Behera, M. Siliwal and G. Sahu (WILD-07-ARA-192, 193, 194); 2 females, 6.viii.2007, Kapilas temple campus, Dhenkanal District, 1944'39.1'N \& 83006'34.8"E, 280m, coll. S. Behera and G. Sahu, (WILD-07-ARA-199, 200); 2 immature, 25.viii.2007, Chipilima, Sambalpur District, $21^{\circ} 21^{\prime} 18.0^{\prime \prime} \mathrm{N} \& 83^{\circ} 55^{\prime} 08.4$ "E, 167m, coll. S. Behera and G. Sahu, (WILD-07-ARA-225,226); 1 immature, 23.viii.2007, Tara Tarini temple grove, Ganjam District, 19029'23.0"N \& 84054'09.8”E, 24m, coll. S. Behera, M. Siliwal and G. Sahu (WILD-07-ARA-240); 1 immature, 20.viii.2007, Sanakuda Island, Chilika Lake, 19033'12.4"N \& 85008'01.7"E, 112m, coll. S. Behera, M. Siliwal and G. Sahu (WILD-07-ARA-243).

Diagnosis: Female resembles $H$. prostans in having unequal bifid tooth on all leg claws and median eyes smaller than lateral eyes. It differs from $H$. prostans in having 4-17 cuspules on labium (Simon 1892; Pocock 1900).

Description of female from Barkuda Island: Total length, 12.72. Carapace 5.68 long, 4.0 wide; chelicerae 2.26 long; abdomen 7.04 long, 4.56 wide. Spinnerets: PMS, 0.72 long, 0.5 wide, 0.26 apart; PLS, 1.0 basal, 0.6 


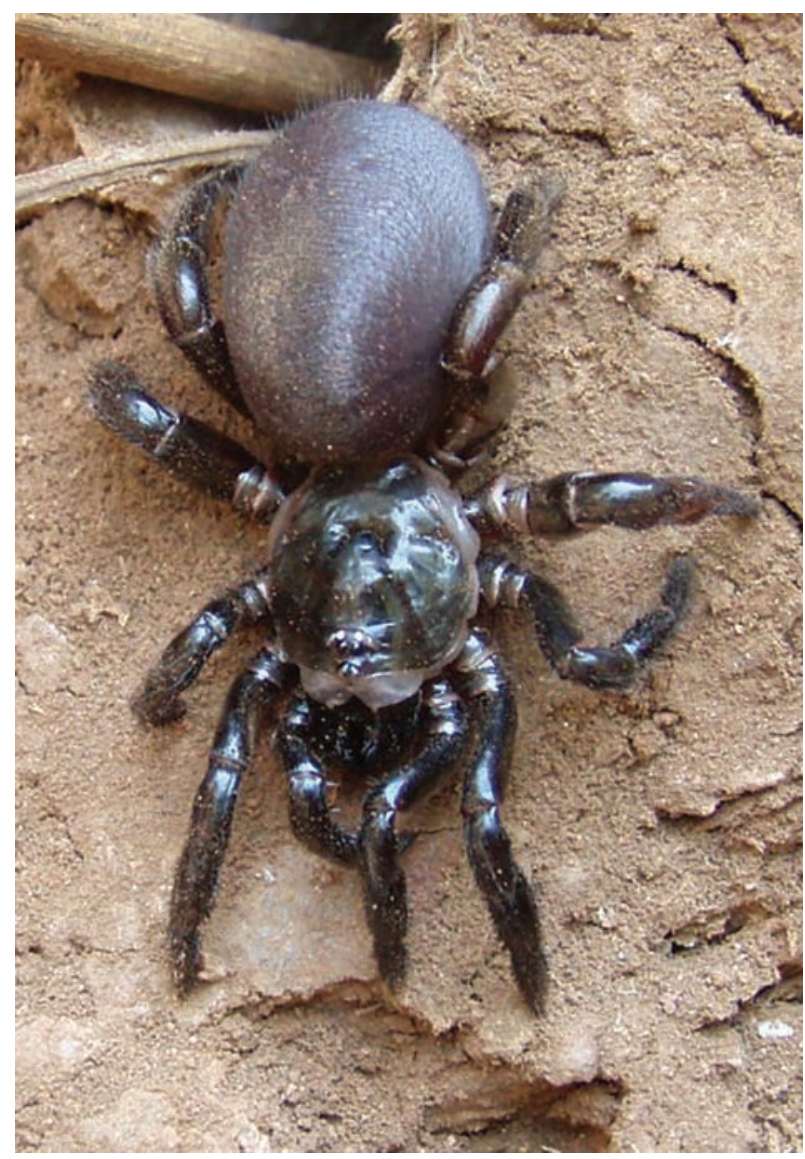

Image 1. Heligmomerus barkudensis in life

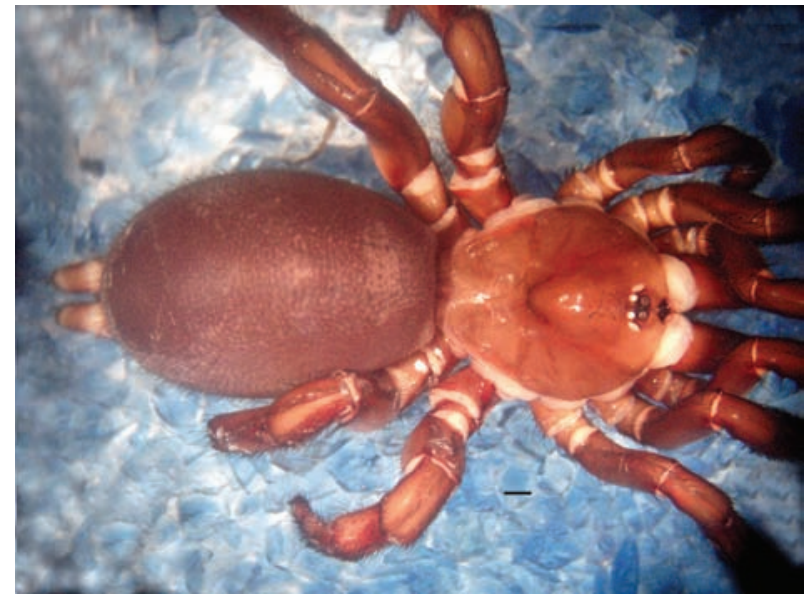

Image 2. Heligmomerus barkudensis, dorsal view. Scale $=1 \mathrm{~mm}$ middle, 0.34 distal; midwidths $0.78,0.6,0.44$ respectively; 1.94 total length.

Colour in life (Image 1): Cephalothroax greenishblack with a darker band running on either side of caput emerging from fovea, legs blackish-green and abdomen uniformly grayish-dark brown.

Colour in alcohol (Image 2): Cephalothorax, chelicerae, maxillae and labium reddish-brown; sternum and coxae yellowish-brown. Abdomen, dorsally brown with small inconspicuous light spots running dorsally to lateral sides in curved lines; ventrally yellowish-green. Legs above brownish-green, darker towards distal segments (tibiae, metatarsi, tarsi); ventral side of legs and palp yellowish-green.

Carapace greenish-brown, glabrous, no hairs except for few bristles on caput, few lines of depression along interstitial ridges and laterally, weak crenulations on caput, more conspicuous near eye group and anteriolaterally, elsewhere absent or negligible. Caput with distinct mound between fovea and eyes, mound as high as ocular tubercle/ eyes (Fig. 2). Fovea deep, procurved, U-shaped. Bristles: one on clypeal edge, caput with four long ones and three long ones between the anterior eyes.

Eyes (Fig. 1, Image 3): Eight in three rows, ALE situated far away from AME on clypeal edge; posterior row procurved. Ocular group 1.2 long, 1.2 wide; MOQ square, 0.6 wide and long. Diameter AME 0.24, PME 0.16, ALE 0.48, PLE 0.34; distance between ALE-AME 0.5, AMEAME 0.05, PLE-PME 0.05, PME-PME 0.6, ALE-PLE 0.8, ALE-ALE adjacent.

Maxillae (Fig. 3, Image 5): 1.6 long anteriorly, 2.2 long posteriorly, 1.2 wide; cuspules ca. 70 , evenly spread over maxillae length; size varies; anterior lobe distinct.

Labium (Fig. 3, Image 5): 1.0 long, 1.26 wide, shallow labiosternal groove present, slightly procurved, four cuspules of varying size in distal 1/3 area.

Chelicerae (Figs. 4-5, Image 4): Five teeth on promarginal and three teeth on retromarginal; a depression on retrolateral face where fang touches chelicerae; rastellum conspicuous, raised on a high triangular mound, consist of 19 thick, short spines at vertical face and up, many normal pointed spines present vertically and dorsally on chelicerae; two hairless bands running over length of dorsal surface of chelicerae.

Sternum (Fig. 3, Image 6): 3.82 long, 3.18 wide, broader between posterior coxae; yellowish-brown, elevated in center, slopping laterally, covered with long black hair; a row of long bristles present on margins, posterior angle blunt but not separating coxae IV.

Sigilla (Fig. 3, Image 6): Posterior sigilla absent; median pair submarginal, 2.18 apart, 0.2 from margin and anterior pair round, marginally.

Legs: Posterior pair of legs thicker than anterior pairs; reddish-brown above and light yellowish-green below, except tarsi of palp and metatarsi and tarsi of all legs that are blackish-brown above and brown below; femora III 


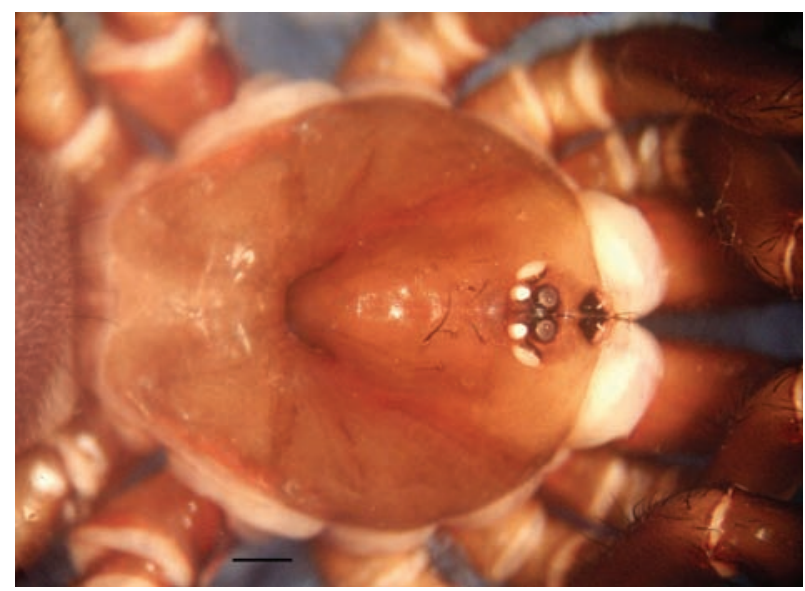

Image 3. Cephalothorax. Scale $=1.0 \mathrm{~mm}$

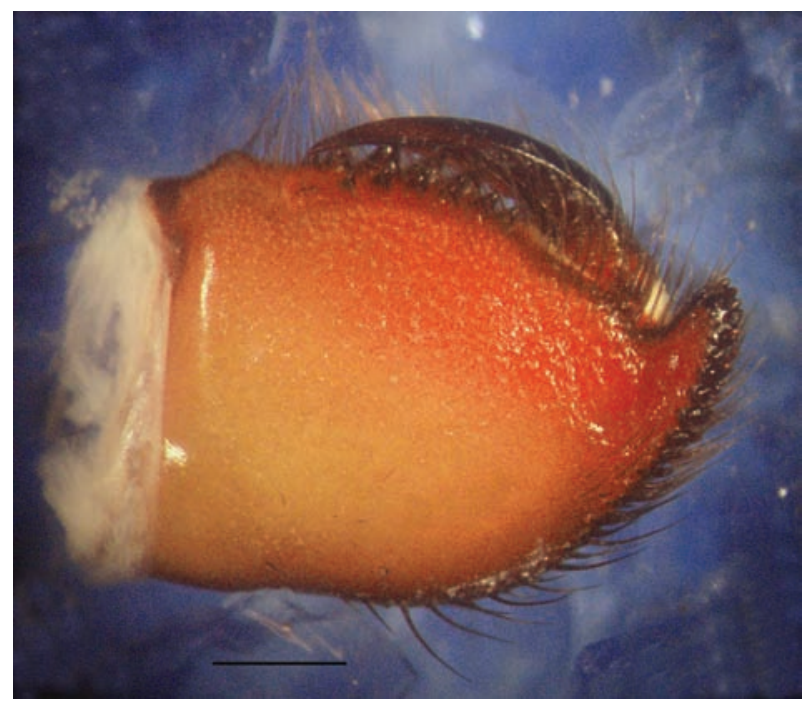

Image 4. Chelicerae rastellum prolateral face. Scale $=1.0 \mathrm{~mm}$

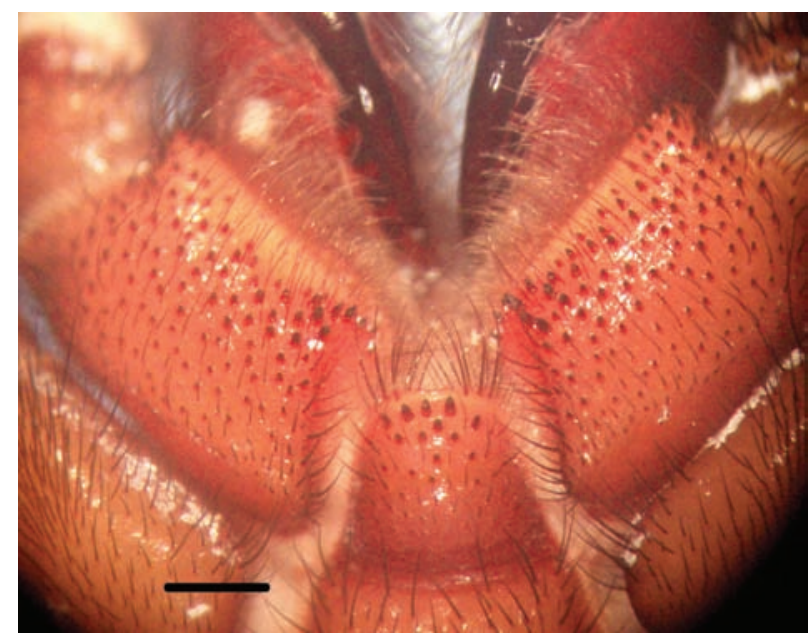

Image 5. Maxillae, labium. Scale $=1.0 \mathrm{~mm}$

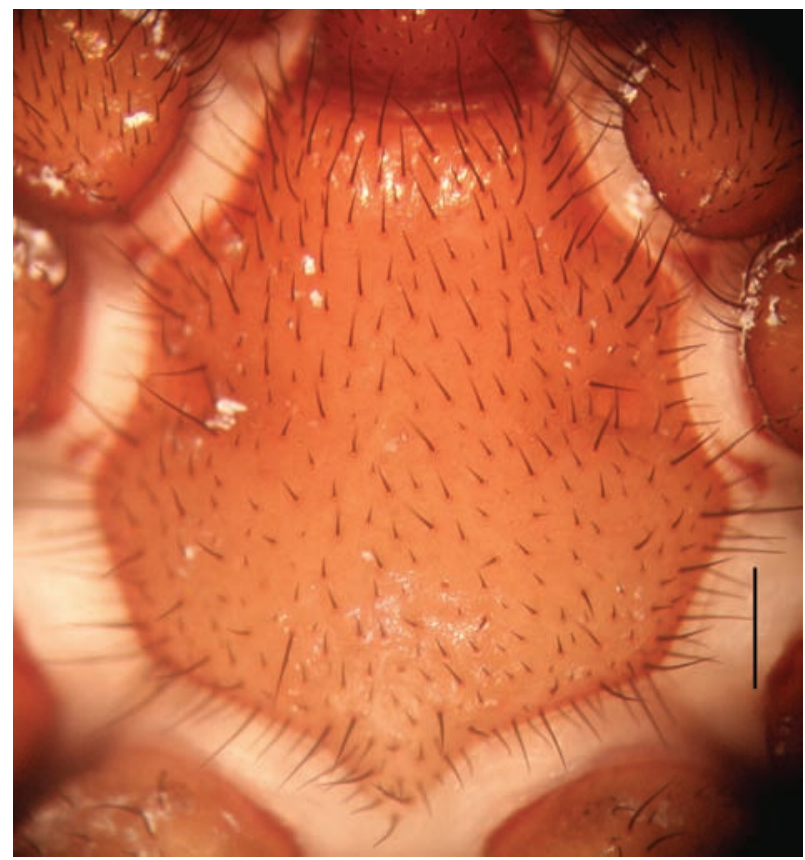

Image 6. Sternum. Scale $=1.0 \mathrm{~mm}$

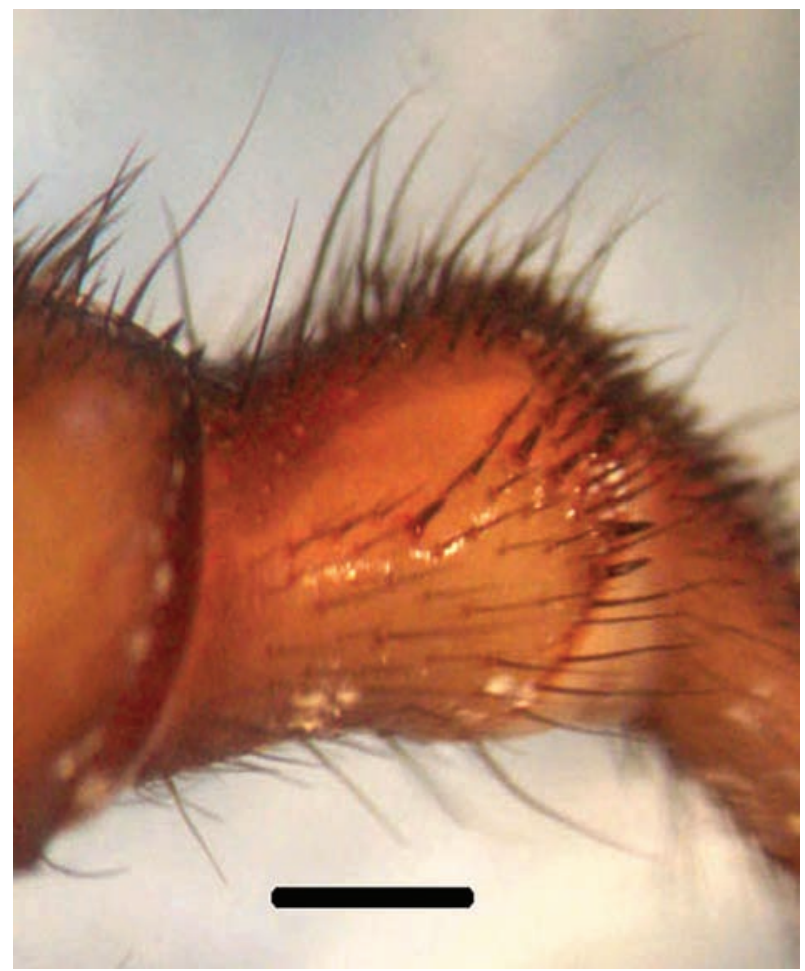

Image 7. Tibia III. Scale $=1.0 \mathrm{~mm}$ 


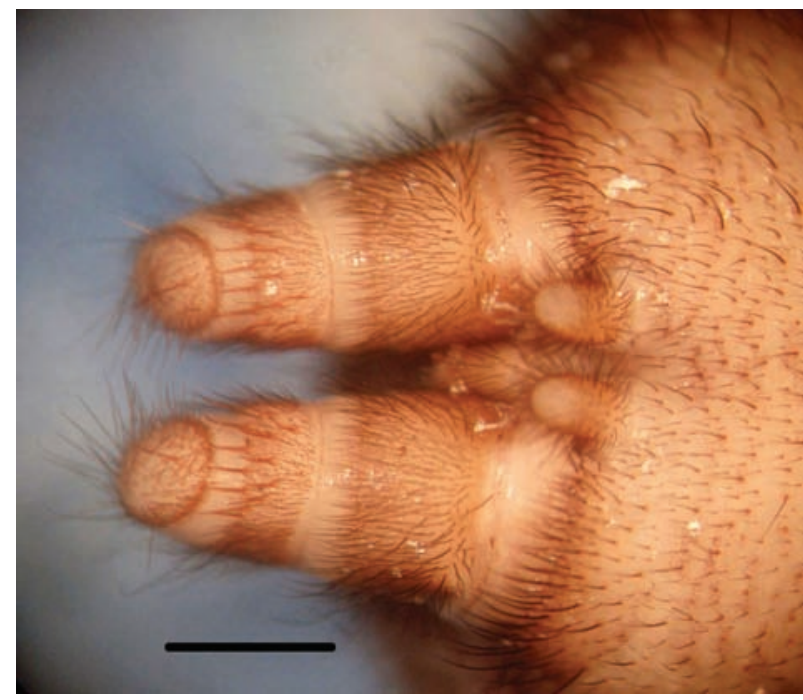

Image 8. Spinnerets. Scale $=1.0 \mathrm{~mm}$

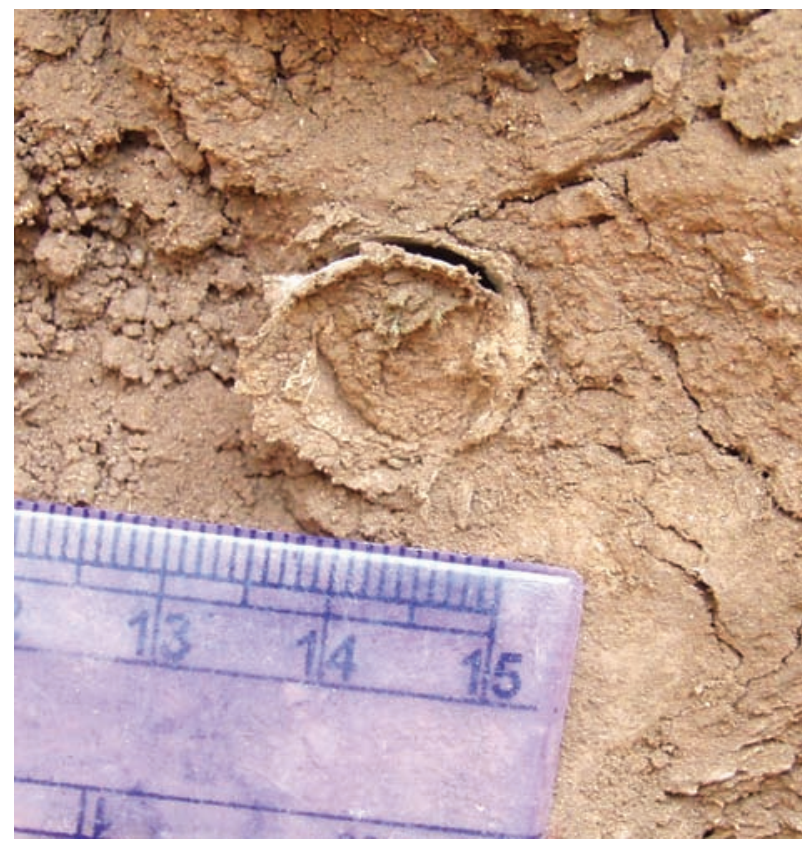

Image 10. Trapdoor nest

clearly wider than rest; metatarsi of all legs longer than tarsi. Tibiae III with saddle-shape depression on basal upper part (Fig. 6, Image 7). Tibiae, metatarsi and tarsi of all legs and tibiae and tarsi of palp dorsoventrally flattened. Coxae of legs yellowish-brown ventrally. Legs covered with few scattered hair, bristles and few curved thick thorn-like spines. Two conspicuous hairless bands running over length of femora, patellae and tibiae. Scopulae absent on tarsi of all legs and palp. Leg formula 4123.

Spines: Curved thick thorn-like spines, leg I: ti, $p=9$, $r=12$; $m t, p=14, r=19$, ta, $p=9, r=8$; leg II: ti, $p=7$ broken, $r=5$ broken, $m t, p=18, r=6$; ta $p=8, r=4$; leg III: $p a, p=23$, $r=4$; ti, $p=21, r=15 ; \mathrm{mt}, \mathrm{p}=14, \mathrm{v}=2$; ta, $v=7$; leg IV: $p$ a $p=50$;

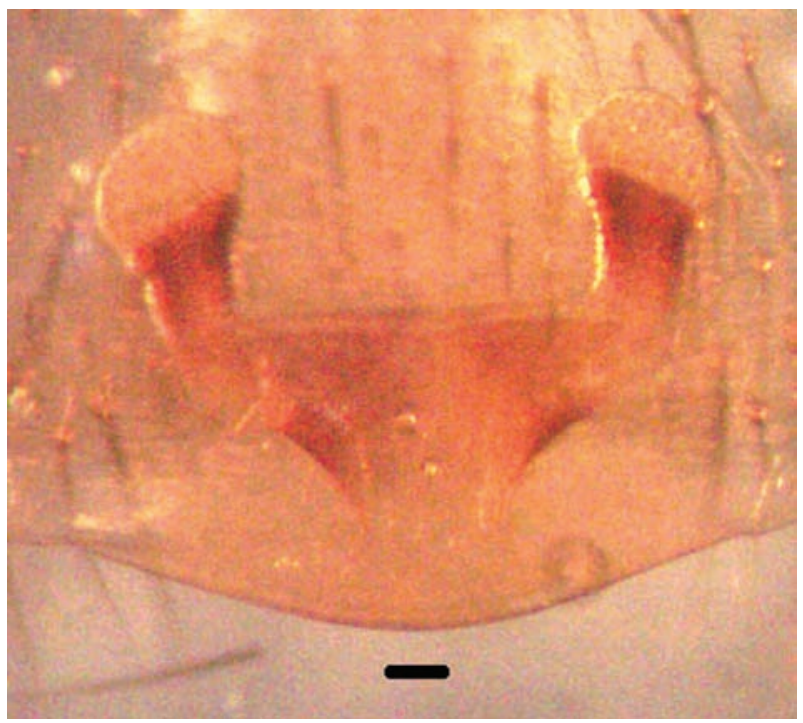

Image 9. Spermethecae. Scale $=0.1 \mathrm{~mm}$

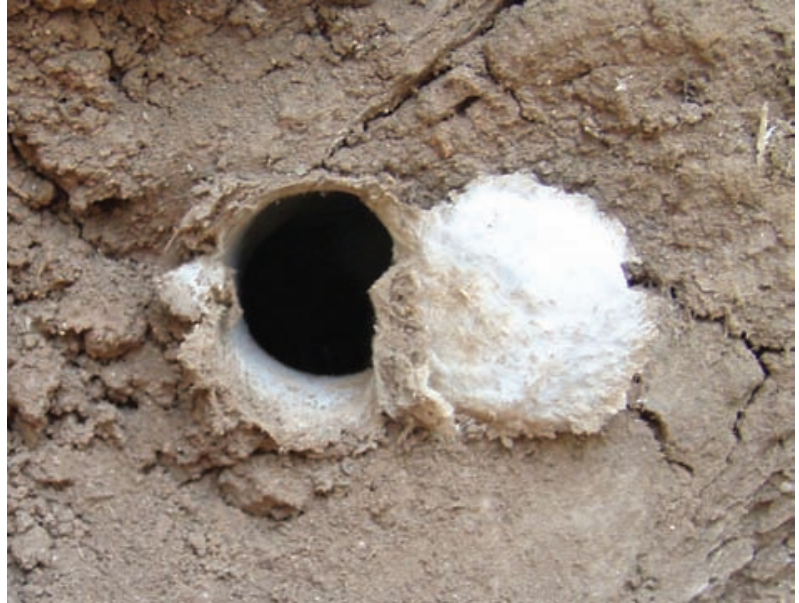

Image 11. Open trapdoor nest

$\mathrm{mt}, \mathrm{v}=5$; ta $\mathrm{v}=151$; palp: ti, $p=r=16$; ta, $p=23, v=3, r=19$.

Trichobotheria: Clavate absent; ta I, 8-10 long filiform in two rows for length; ta II, 10-12 long filiform in two rows for length; ta III, 12-14 long filiform in basal two third; ta IV, 10-12 long filiform in distal three fourth. Mt I, 10-12 long filiform in distal one-fourth; mt II-IV, 5 long filiform in distal one-fourth.

Leg coxae: Coxa I covered with long black bristles in distal half, rest with short and long spinules. Coxa II covered with short spinules and long bristles. Coxa III with a central patch without hair or spinules, rest sparsely covered with spinules and long bristles. Coxa IV clearly broader than rest, anterior edge curved, sparsely covered with spinules and long bristles.

Claws (Figs. 7-8): All legs with three claws, paired claw with single unequal bifid tooth. Palp, single claw with unequal bifid tooth. Claw tufts absent.

Abdomen (Fig. 1, Image 1-2): Grayish brown, dorsum with many faint cream spots radiating in curved 

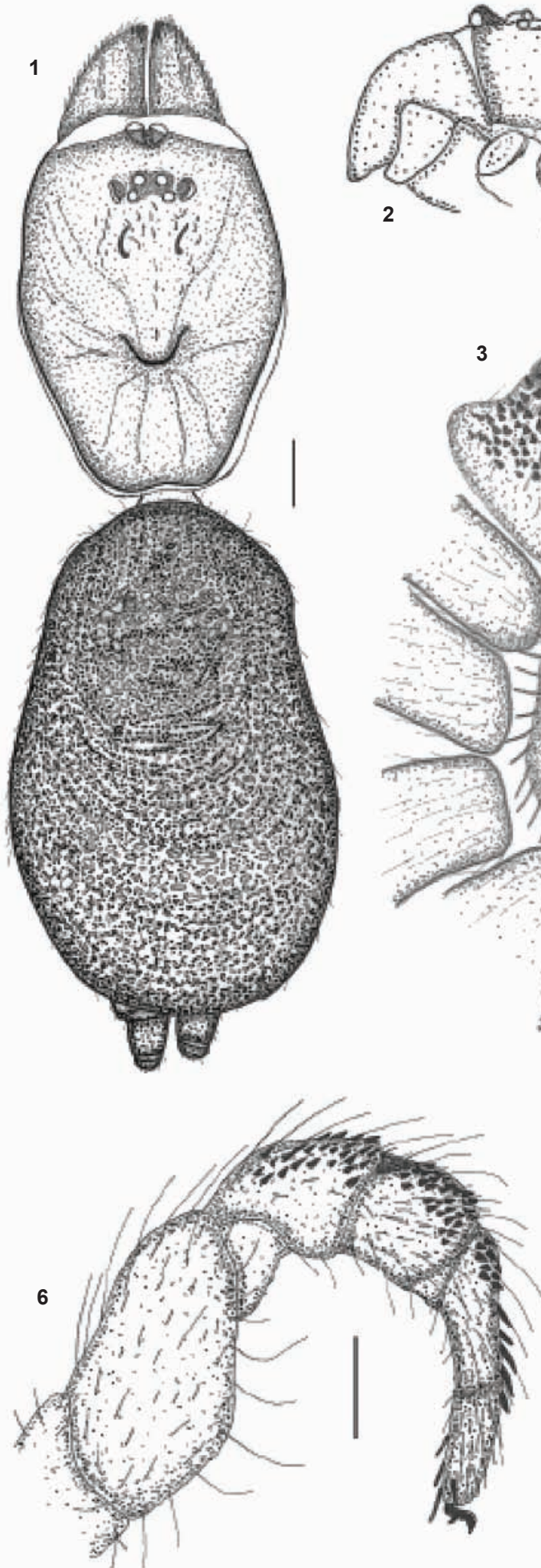
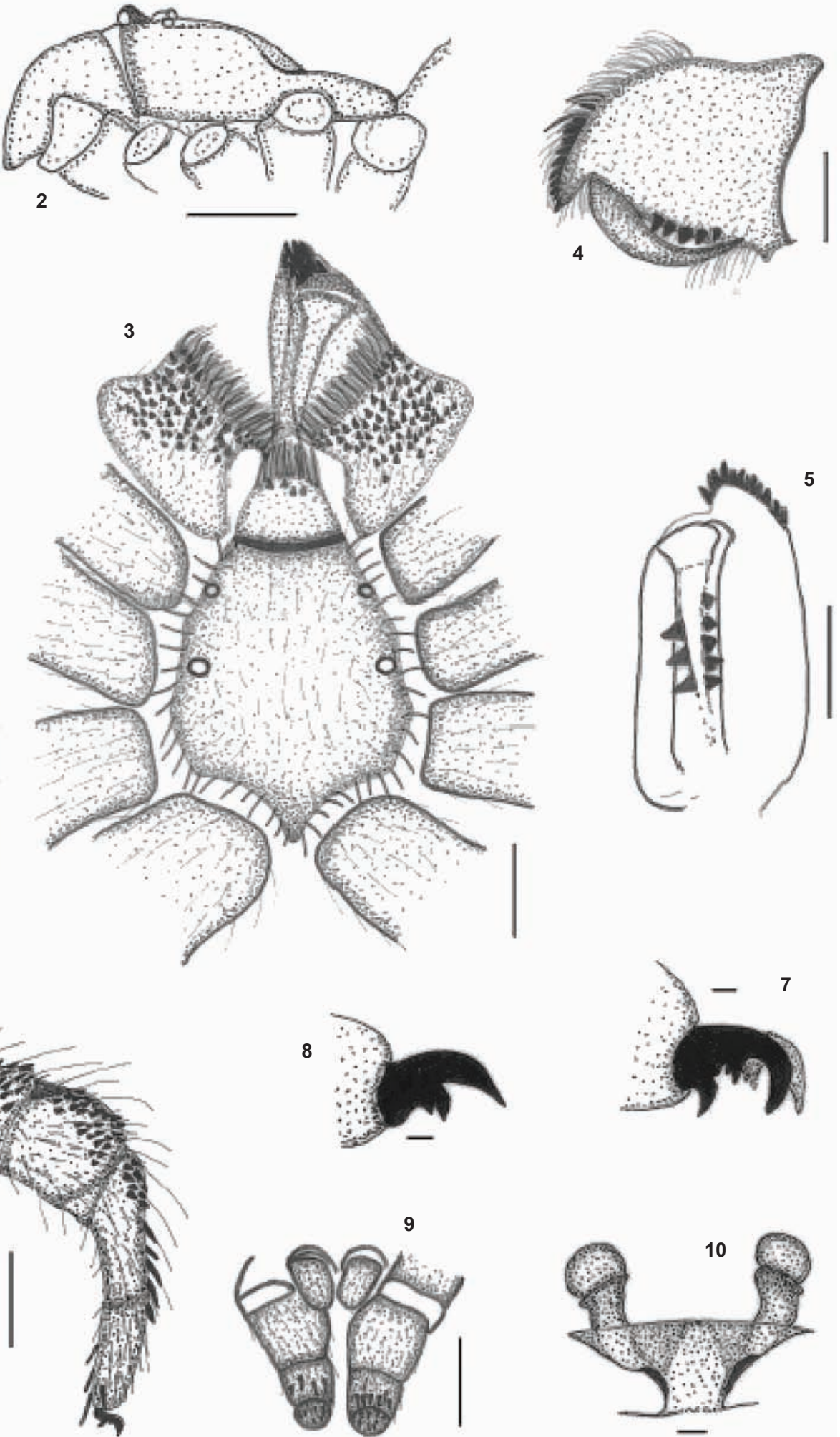

Figure 1-10. Heligmomerus barkudensis (Gravely, 1921), female. Scale $=1.0 \mathrm{~mm}$.

1 - Cephalothorax andabdomen, dorsal view; 2 - Cephalothorax, lateral view; 3 - Sternum, labium, maxillae and chelicerae; 4 - Chelicerae prolateral view; 5 - Chelicerae, marginal teeth; 6 - Leg III (Femur to Tarsus) retrolateral view; 7 - Claw of leg I $($ scale $=0.1 \mathrm{~mm}) ; 8$ - Claw of palp $($ scale $=0.1 \mathrm{~mm}) ; 9-$ Spinnerets; $10-$ Spermethecae $($ scale $=0.1 \mathrm{~mm})$. 


\begin{tabular}{|c|c|c|c|c|c|c|c|c|c|c|}
\hline & 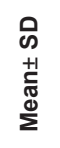 & $\begin{array}{l}\mathscr{2} \\
\circ \\
0 \\
+ \\
0 \\
\circ \\
\dot{+}\end{array}$ & $\begin{array}{l}\text { No } \\
0 \\
+1 \\
\infty \\
\infty \\
\stackrel{N}{N}\end{array}$ & $\begin{array}{l}\overline{0} \\
0 \\
+1 \\
\hat{+} \\
\stackrel{N}{0}\end{array}$ & , & 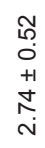 & 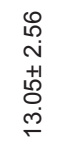 & & 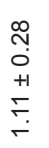 & 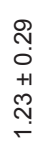 \\
\hline \multirow[t]{2}{*}{$\frac{2}{\bar{c}}$} & 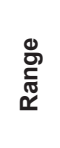 & 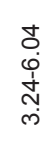 & 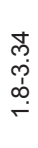 & 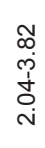 & , & 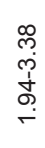 & $\begin{array}{l}\infty \\
\infty \\
\infty \\
\stackrel{0}{0} \\
\dot{\tilde{\sigma}} \\
\dot{\sigma}\end{array}$ & & 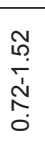 & 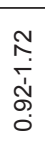 \\
\hline & $\stackrel{*}{\sigma}$ & $\underset{\sim}{\stackrel{\sim}{\sim}}$ & $\stackrel{\circ}{\stackrel{\circ}{\leftarrow}}$ & $\underset{\sim}{\stackrel{d}{N}}$ & , & $\stackrel{\stackrel{\leftrightarrow}{\circ}}{\circ}$ & $\begin{array}{l}\tilde{\sigma} \\
\stackrel{\sigma}{0}\end{array}$ & & N̂ & $\stackrel{\circ}{\stackrel{r}{r}}$ \\
\hline \multirow{3}{*}{$\geq$} & 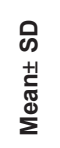 & $\begin{array}{l}\stackrel{\sim}{1} \\
+1 \\
\stackrel{+}{0} \\
i\end{array}$ & 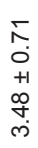 & $\begin{array}{l}\tilde{N} \\
\delta \\
0 \\
+1 \\
\infty \\
\infty \\
\infty\end{array}$ & 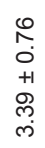 & 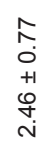 & $\begin{array}{l}\infty \\
\stackrel{2}{0} \\
\dot{+} \\
+1 \\
\stackrel{+}{\infty} \\
\infty \\
\infty\end{array}$ & & 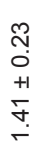 & 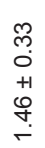 \\
\hline & $\begin{array}{l}\mathscr{D} \\
\text { : } \\
\text { of }\end{array}$ & 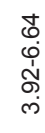 & 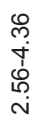 & 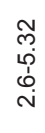 & 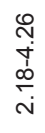 & 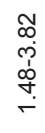 & 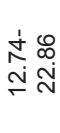 & & 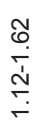 & 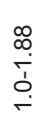 \\
\hline & $\stackrel{*}{\sigma}$ & $\underset{\widetilde{\sigma}}{\widetilde{\infty}}$ & $\stackrel{\stackrel{\leftrightarrow}{N}}{\stackrel{N}{N}}$ & $\stackrel{\leftrightarrow}{\stackrel{\sim}{\sim}}$ & $\stackrel{\infty}{\stackrel{N}{N}}$ & 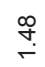 & 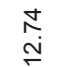 & & 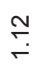 & $\stackrel{8}{\circ}$ \\
\hline \multirow{3}{*}{ 胥 } & 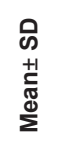 & $\begin{array}{l}\infty \\
\stackrel{\infty}{0} \\
+ \\
+1 \\
\infty \\
\infty \\
\infty\end{array}$ & $\begin{array}{l}\infty \\
\infty \\
0 \\
+1 \\
+1 \\
\stackrel{0}{o} \\
\stackrel{N}{N}\end{array}$ & $\begin{array}{l}\text { I } \\
\text { o } \\
+1 \\
\hat{O} \\
\text { i }\end{array}$ & 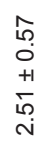 & $\begin{array}{l}\stackrel{+}{0} \\
0 \\
+ \\
+ \\
\& \\
\stackrel{+}{+}\end{array}$ & 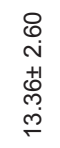 & & 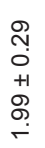 & 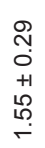 \\
\hline & 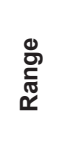 & 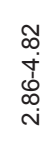 & $\begin{array}{l}\stackrel{N}{\sim} \\
\stackrel{\sim}{\sim} \\
\stackrel{\sim}{\sim}\end{array}$ & 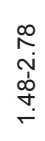 & 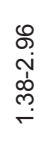 & 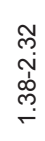 & 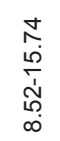 & & 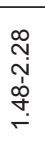 & 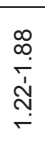 \\
\hline & $\stackrel{*}{\stackrel{*}{\sigma}}$ & $\begin{array}{l}\circ \\
\stackrel{\infty}{N} \\
\end{array}$ & $\stackrel{\text { f }}{\sim}$ & 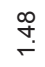 & $\stackrel{\infty}{\stackrel{\infty}{\sim}}$ & $\stackrel{\infty}{\stackrel{\infty}{\sim}}$ & $\begin{array}{l}\mathbb{N} \\
\text { No } \\
\infty\end{array}$ & & 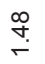 & $\underset{\sim}{\mathbb{N}}$ \\
\hline \multirow{3}{*}{ 㷰 } & 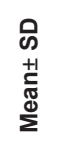 & 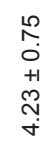 & $\begin{array}{l}0 \\
\text { L } \\
0 \\
+1 \\
+1 \\
\stackrel{N}{N} \\
\stackrel{N}{N}\end{array}$ & $\begin{array}{l}\text { f. } \\
0 \\
+1 \\
0 \\
0 \\
\text { i }\end{array}$ & $\begin{array}{l}\text { @o } \\
0 \\
+1 \\
+1 \\
\hat{i} \\
\text {. }\end{array}$ & 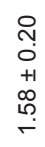 & 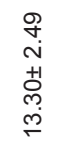 & & 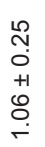 & 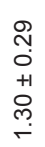 \\
\hline & 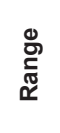 & $\begin{array}{l}\text { iे } \\
\text { ధें } \\
\dot{m}\end{array}$ & 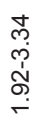 & 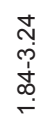 & 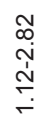 & $\begin{array}{l}\stackrel{\circ}{\infty} \\
\stackrel{+}{\infty} \\
\stackrel{\infty}{\leftarrow}\end{array}$ & 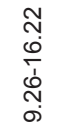 & & 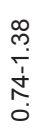 & 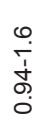 \\
\hline & $\stackrel{*}{\sigma}$ & 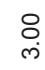 & $\underset{\sim}{\stackrel{\alpha}{\sigma}}$ & $\stackrel{+\infty}{\stackrel{+}{r}}$ & $\stackrel{\sim}{\check{r}}$ & $\stackrel{\infty}{\stackrel{\infty}{\sim}}$ & 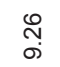 & & 范 & $\begin{array}{l}\text { t. } \\
\text { ò }\end{array}$ \\
\hline \multirow{3}{*}{$\bar{\Xi}$} & 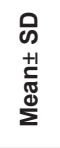 & 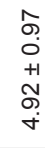 & $\begin{array}{l}8 \\
0 \\
0 \\
+1 \\
\infty \\
\infty \\
\dot{m}\end{array}$ & 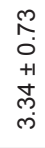 & $\begin{array}{l}\text { No } \\
\text { O } \\
+1 \\
\stackrel{N}{N} \\
\text { N }\end{array}$ & 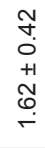 & 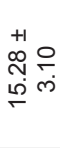 & & 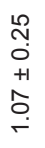 & 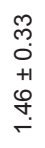 \\
\hline & 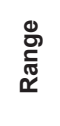 & $\begin{array}{l}\text { N } \\
0 \\
\vdots \\
0 \\
\dot{j}\end{array}$ & 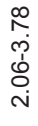 & 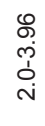 & $\begin{array}{l}0 \\
\text { गें } \\
\stackrel{\leftrightarrow}{0}\end{array}$ & 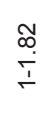 & 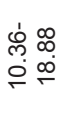 & & 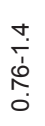 & 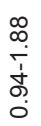 \\
\hline & $\stackrel{*}{*}$ & $\underset{\text { d }}{\stackrel{్}{~}}$ & 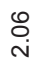 & $\underset{\sim}{\stackrel{\sim}{\sim}}$ & $\stackrel{\leftrightarrow}{\check{r}}$ & $\stackrel{8}{r}$ & $\begin{array}{l}\stackrel{0}{e} \\
\stackrel{0}{0}\end{array}$ & & $\begin{array}{l}\stackrel{0}{2} \\
\stackrel{0}{0}\end{array}$ & 苟 \\
\hline & & 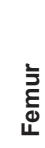 & $\begin{array}{l}\overline{\bar{\Phi}} \\
\text { sँ }\end{array}$ & 彦 & 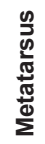 & 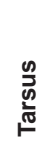 & $\begin{array}{l}\overline{\text { गू }} \\
\stackrel{\circ}{\circ}\end{array}$ & $\frac{\sum_{0}^{0}}{\frac{3}{2}}$ & 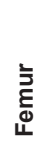 & 荡 \\
\hline
\end{tabular}


line; covered with short black hair with few long bristles mid dorsally, cuticle appears leathery and a little rough. Ventrally yellowish-green, uniformly covered with short and long black hairs.

Spinnerets (Fig. 9, Image 8): PMS digitiform covered with brown hair; PLS covered with brown hair, apical segment dome-shaped.

Spermathecae (Fig. 10, Image 9): A pair of spermathecae, emerging from anterior ends of a triangular plat like sclerotized structure, apical ends of spermathecae rounded like a mushroom placed on a stalk.

Variations: Specimen from Barkuda Island is much smaller than the specimens collected from various locations on mainland in Orissa. Total length 12.72-19.34 (17.25 \pm 2.55$)$. Carapace 5.68-8.7 (7.37 \pm 1.05$)$ long, 4.07.68 (6.34 \pm 1.34$)$; chelicerae 2.26-4.38 (3.63 \pm 0.8$)$ long. Sternum: 3.82-6.0 (4.8 \pm 0.87$)$ long, 3.18-5.04 (4.17 \pm 0.7$)$ wide. Labium: 1.0-1.52 (1.26 \pm 0.2$)$ long, 1.26-2.0 $(1.61 \pm 0.33)$ wide, cuspules 4-17 $(12.33 \pm 4.5)$. Maxillae: 1.6-2.68 (1.95 \pm 0.38$)$ long in front, 2.2-3.92 $(3.03 \pm 0.57)$ long in back, 1.2-2.4 (1.86 \pm 0.46$)$ wide; cuspules ca. 70-100 (80.0 \pm 12.65$)$. Abdomen 7.04-11.8 (9.88 \pm 1.69$)$ long, 4.56-8.02(6.74 \pm 1.42$)$ wide. Spinnerets: PMS, 0.72$1.26(1.0 \pm 0.18)$ long, $0.4-0.68(0.53 \pm 0.11)$ wide, 0.26 $0.7(0.45 \pm 0.18)$ apart; PLS, 0.98-1.82 (1.33 \pm 0.33$)$ basal, 0.5-0.8 (0.64 \pm 0.12$)$ middle, 0.34-0.8 (0.55 \pm 0.2$)$ distal;

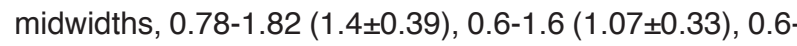
$1.1(0.82 \pm 0.26)$ respectively; $1.94-2.98(2.58 \pm 0.41)$ total length.

Male: Unknown.

\section{Distribution: Orissa, India}

\section{Natural History}

The forest from where these spiders were collected was primarily of dry or moist deciduous type. Specimens were also found in plantations and in degraded secondary scrubs as observed on the islands of Chilika. Spider burrows were mainly found on roadside bunds, either in open slopes or with partially overhanging vegetation. On the islands, burrows were also located at the base of trees or shrubs, or between rocks, and at times on termite mounds.

The spider burrows were simple, tubular, with a thick lining of silk, measuring 35-60 mm in length and 12-15 $\mathrm{mm}$ in diameter (Images 10-11). The entrance to the burrow had a single hinged door, which was round and measured 16-18 mm in diameter. The entrance door on the outer side was usually covered with mosses, leaf and or soil particles, while on the inner side it was lined with silk which was slightly thicker towards the center than along the edges.

\section{REFERENCES}

Cambridge, O.P-. (1890). On some new species and two new genera of Araneida. Proceedings of the Zoological Society of London 1890: 620-629.

Dippenaar-Schoeman, A.S. (2002). Baboon and Trapdoor spiders of Southern Africa: An Identification Manual. Plant Protection Research Institute Handbook No. 13, Agriculture Reseach Council, Pretoria, 128pp.

Gravely, F.H. (1915). Notes on Indian mygalomorph spiders. Records of the Indian Museum, Calcutta 11: 257-287.

Gravely, F.H. (1921). The spiders and scorpions of Barkuda Island. Records of the Indian Museum, Calcutta 22: 399421.

Gravely, F.H. (1935). Notes on Indian mygalomorph spiders. II. Records of the Indian Museum, Calcutta 37: 69-84.

Platnick, N.I. (2010). The world spider catalog, version 10.5 . American Museum of Natural History, online at http:// research.amnh.org/entomology/spiders/catalog/index.html. Downloaded on 25 February 2010.

Pocock, R.I. (1900). The Fauna of British India, Including Ceylon and Burma - Arachnida. London, 279pp.

Raven, R.J. (1985). The spider infraorder Mygalomorphae (Araneae): Cladistics and systematics. Bulletin of the American Museum of Natural History 182: 1-180.

Siliwal, M. \& S. Molur (2007). Checklist of spiders (Arachnida: Araneae) of South Asia including 2006 update of Indian spider checklist. Zoos' Print Journal 22(2): 2551-2597+ i-lxxxiv (web supplement).

Siliwal, M., S. Molur \& B.K. Biswas (2005). Indian spiders (Arachnida: Araneae): updated checklist 2005. Zoos' Print Journal 20(10): 1999-2049.

Simon, E. (1892). Histoire naturelle des araignées. Paris, 1: $1-256$.

Author Contribution: MS carried out surveys in Orissa and taxonomy of the paper. Identification and description of the species was done by MS with the help of RR in this paper. RR and SM contributed in editing the paper.

Acknowledgements: The authors are grateful to the following personnel/ Organisations: Sally Walker, Zoo Outreach Organisation for her constant support to the Indian Tarantula project; PCCF and Dr. S.K. Kar, Orissa Forest Department for giving permission to carry out spider surveys in different protected areas in Orissa; DEFRA / FFI Flagship Species Fund (project No. 06/16/02 FLAG) for financial support to this part of Indian Tarantula project; Suresh Kumar, Wildlife Institute of India, for commenting on the first draft of this paper; Saroj Behera and Ganapati Sahu, for their assistance during field work; and Prof. M. Ganeshkumar, Tamil Nadu Agriculture University, Coimbatore, for providing technical support. 